\title{
The Angantyr Model for Heavy-Ion Physics in PYTHIA8
}

\author{
Harsh Shah \\ Department of Astronomy and Theoretical Physics, Solvegatan 14A, S-223 62 Lund, Sweden; \\ harsh.shah@thep.lu.se \\ + Presented at Hot Quarks 2018-Workshop for Young Scientists on the Physics of Ultrarelativistic \\ Nucleus-Nucleus Collisions, Texel, The Netherlands, 7-14 September 2018. \\ $\ddagger$ LU-TP 18-37.
}

Published: 12 April 2019

\begin{abstract}
We have formulated a new model for collisions with nuclei, called Angantyr, which is now included in PYTHIA8 event generator. The model is inspired by the old Fritiof model, but also includes effects of hard partonic interactions. It uses a Glauber model to calculate the number of wounded nucleons, but includes fluctuations in the nucleon-nucleon(NN) interaction to separate non-diffractively and diffractively wounded nucleons. The MC simulates final states without assuming a thermalised plasma. In this manner we are providing an event generator to be used to simulate events from pp to AA with the same underlying physics approach. Collective effects due to high string density are not included in the present version, but will be added in future work.
\end{abstract}

Keywords: PYTHIA8; heavy ion collisions

\section{Introduction}

Recently several features exclusive to heavy ion physics have also been observed in pp collisions at the LHC. Observations of strangeness enhancement [1] as well as ridge [2] like shapes in pp collision results created turmoil in the particle physics community. These observations require investigation of similarities between pp and heavy ion collisions through their final state particle production and distribution. In this proceeding paper we provide an overview of our initiative to approach this from pp collision side and attempt to extrapolate our knowledge from smaller systems to heavy ions.

Experimental results for pp collisions are usually well explained by event generators like SHERPA [3], Herwig [4] and PYTHIA8 [5]. Heavy ion collision results are usually compared with the study specific event generators like EPOS-LHC [6], AMPT [7] and HIJING [8]. Our model "Angantyr" is developed within PYTHIA8 event generator. It can reproduce general features of pA and AA collisions without being tuned to any heavy ion data. The model generates events without assuming production of the thermalised medium after the collision. Hence, the current version of the model can be used to study non-collective effects.

\section{Overview of the Event Generation}

Some major modifications are introduced in the extrapolation of pp collision dynamics of PYTHIA8 event generator. The Angantyr model improves the inclusive definition of collision types of the old Fritiof model $([9,10])$. In heavy ion collisions a projectile nucleon can interact with multiple target nucleons. Among these possible interactions there will be only one primary collision which will look like a standard pp none-diffractive (ND) collision. But multiple other target nucleons can also show possibility of ND interaction with the projectile nucleon. We consider these interactions as secondary ND. In the Angantyr model we performed a careful analysis to accommodate these 
secondary ND collisions in heavy ions as modified single-diffractive (SD) interactions. The SD in pp is treated as interaction between a pomeron and a proton in PYTHIA8 event generator. To distinguish usual SD collisions from secondary ND collisions for the later case we modified the pomeron parton distribution function (PDF) to look like a proton PDF.

In the "Angantyr" model we consider fluctuating wavefunctions of nucleons. We treat them as fluctuations in the nuclei radius. In the impact parameter space interaction cross sections are then interpreted as probabilities. In this way initial state fluctuations are accommodated in our model. For every nucleus-nucleus collision nucleons are distributed randomly inside a nucleus according to a Glauber formalism inspired from work by Strikman et al. [11]. All nucleons are then identified as either wounded or spectators. Depending upon the interaction probability interections between wounded nucleons in projectile and target are marked as elastic, ND, secondary ND, SD and double-diffrative (DD). Further details of these modifications and their implications are described in [12].

All sub-events are then generated as corresponding PYTHIA8 events and at the parton level they are all stacked together to represent one pA or AA collision event. The model always checks for energy momentum conservation while generating secondary events. Hence, in the default set-up we put a constrain over secondary event reconstruction. In the default set-up, the model performs only one attempt to generate a secondary ND collision. The secondary ND is vetoed upon failed attempt for that particular sub-event. In Figure 1a we show that with the default constrain on regenerating secondary ND events in pA collision we quantitatively reproduce the transverse energy $\left(\sum E_{\perp}^{P b}\right)$ distribution.

In central AA collisions a higher number of nucleon-nucleon interactions averages out effects of fluctuations. In such cases majority of interactions are treated as standard ND and they are less dependent on secondary ND events. In [12] we dedicated a section describing effects of our modified $\mathrm{SD}$ events in generating heavy ion collisions.
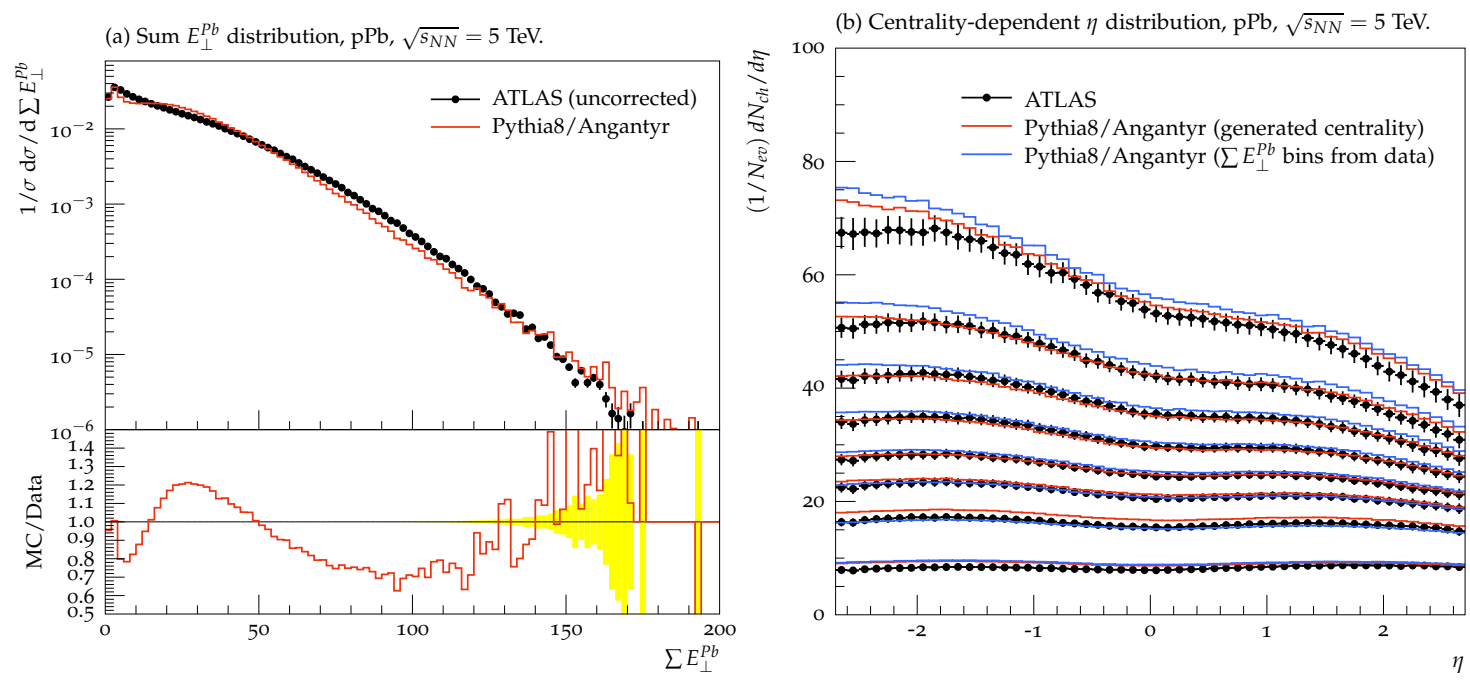

Figure 1. (a) $\sum E_{\perp}^{P b}$ distribution of generated pPb events at $\sqrt{s_{N N}}=5 \mathrm{TeV}$. (b) Charged multiplicity distribution of $\mathrm{pPb}$ collisions at $\sqrt{s_{N N}}=5 \mathrm{TeV}$ from ATLAS [13] compared with the Angantyr generated events with two different means of centrality selection.

\section{Results}

We show comparisons of our model-generated events with experiments such as ATLAS and ALICE for $\mathrm{pA}$ and AA collisions. The results shown here are generated using the default set-up of the "Angantyr" model with PYTHIA8 version 8.235.

\section{1. $p$ A Comparison}

The study of pA interactions is a good stepping stone while extrapolating a pp collision dynamics. In heavy ion collisions events are studied and compared in different bins of event centralities. 
The centrality of an event indirectly defines collision geometry and impact parameter of the interaction. For appropriate comparison we require to obtain model-generated results in the centrality defined by the experiment.

For pA comparison we use centrality definition from $\sum E_{T}$, where $\sum E_{T}$ is the deposited transverse energy in a pseudo-rapidity interval of $\eta=[-4.9,-3.2]$ by the ATLAS experiment [13]. This pseudo-rapidity range is in the direction of $\mathrm{Pb}$ nucleus in $\mathrm{pPb}$ collisions. In Figure 1 a we show $\sum E_{\perp}^{P b}$ distribution for $\mathrm{pPb}$ collisions. The simulated events reproduce $\sum E_{\perp}^{P b}$ distribution fairly well. Figure $1 \mathrm{~b}$ shows average charged particles multiplicity distribution in different centralities. The two solid colour lines in the figure represent event selection by centrality binning based on model-generated centrality (red line) and centrality bins obtained from the data (blue line). For most of the centrality bins our model-generated charged multiplicity distribution quantitatively agrees with the data. Our model over estimates the charged multiplicity for the most (0-5\%) central collisions.

\subsection{AA Comparison}

For AA collisions we compared our results with the ALICE experiment data. Therefor we also require to use centrality as defined in the ALICE experiment [14]. Here, amplitude distribution in forward and backward V0 detectors as well as a trigger set-up is used to define the event centrality. Since, in the event-generator we do not have any direct way to reproduce amplitude distribution of V0 detectors, we used an indirect way to define centrality [12] to compare our results with ALICE data.

In Figure $2 \mathrm{a}$ we show charged multiplicity in the central pseudo-rapidity bin for $\mathrm{PbPb}$ collisions compared with the ALICE data [14] at $\sqrt{s_{N N}}=2.76 \mathrm{TeV}$. In the same figure we also show results from $\sqrt{s_{N N}}=5.44 \mathrm{TeV}$ XeXe collisions [15]. The dashed red line shows the distribution with the ATLAS centrality definition for $\mathrm{PbPb}$ data. Since the solid red line overlaps with the dashed red line it is not distinctively visible in the histogram.
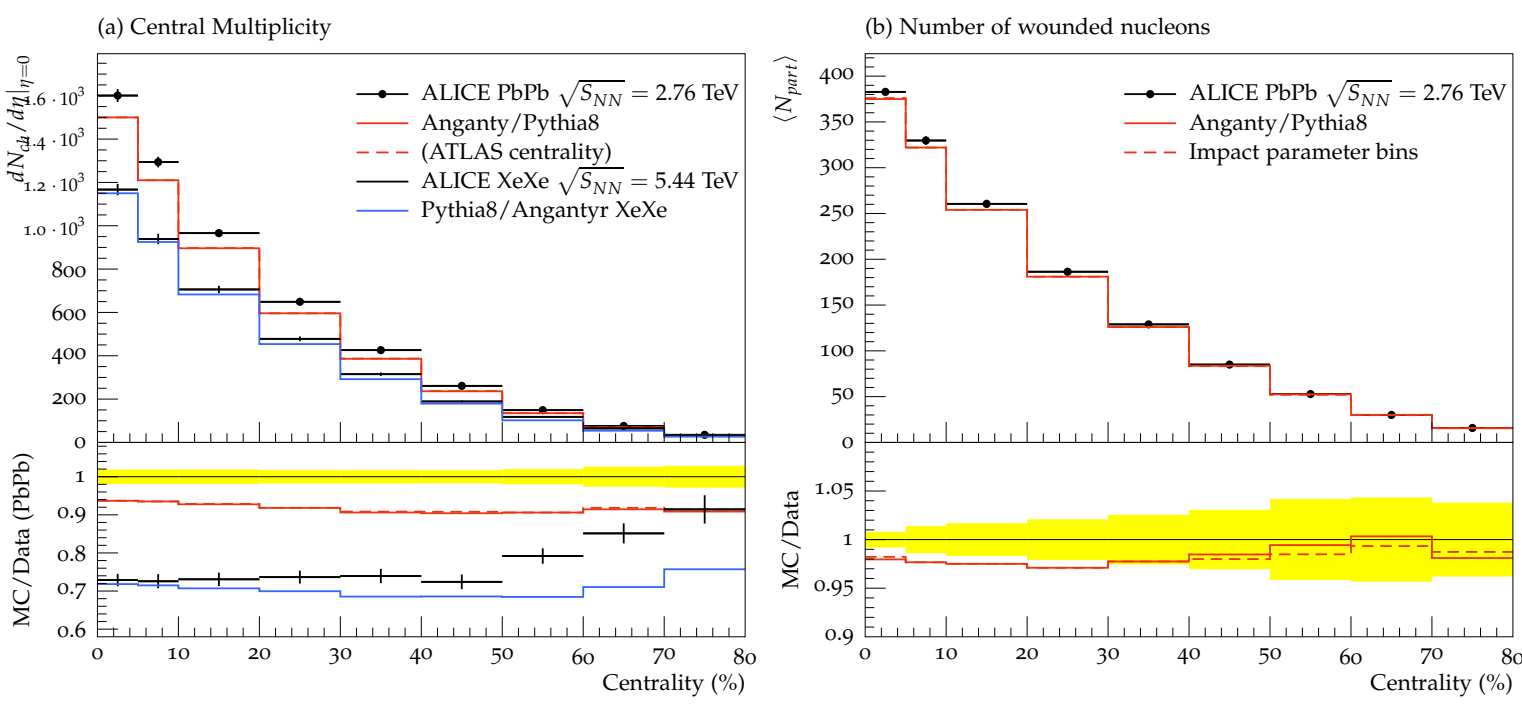

Figure 2. (a) Charged multiplicity at mid-rapidity for $\mathrm{PbPb}\left(\sqrt{s_{N N}}=2.76 \mathrm{TeV}\right)[14]$ and $\mathrm{XeXe}\left(\sqrt{s_{N N}}=5.44\right.$ $\mathrm{TeV}$ ) [15] compared to Angantyr for various centrality. (b) $\left\langle N_{\text {part }}\right\rangle$ as a function of centrality for the Angantyr model compared to $\mathrm{PbPb}\left(\sqrt{s_{N N}}=2.76 \mathrm{TeV}\right)$ Glauber-model calculations from ALICE [14].

For both types of nuclei the Angantyr results quantitatively agree with the experimental data. In Figure $2 b$ the average number of participating nucleons is shown for ALICE PbPb data of Figure $2 a$. The model-generated distribution agrees with data within $5 \%$ discrepancy. The dashed red line in Figure $2 \mathrm{~b}$ shows the number of wounded nucleons obtained as percentiles in the model-generated impact parameter bins. 


\section{Conclusions}

In the "Angantyr" model, unlike the old Fritiof model we separately treat diffractive and non-diffractive excitations. Moreover, the model provides initial state fluctuations in nuclei geometry while sampling nucleons in projectile and target for heavy ion collisions. Our model can provide a quantitative description of the data from pA and AA collisions. A comparison of our model with currently available other models is done in [12].

The current version of our model uses PYTHIA8 for event final state distribution. In PYTHIA8 hadrons are produced via string breaking, where all strings break into hadrons independently. Therefore, the model cannot describe the collective effect and strangeness enhancement in its current version. Despite these limitations, our model can be used as a baseline in the study of collective effects. In coming publications we are keen to focus on these shortcomings of our model.

Funding: Work supported in part by the Swedish Research Council, contracts number 2016-03291, 2016-05996 and 2017-0034, in part by the European Research Council (ERC) under the European Union's Horizon 2020 research and innovation programme, grant agreement No 668679, and in part by the MCnetITN3 H2020 Marie Curie Initial Training Network, contract 722104.

\section{References}

1. ALICE Collaboration. Enhanced production of multi-strange hadrons in high-multiplicity proton-proton collisions. Nature Phys. 2017, 13, 535-539, doi:10.1038/nphys411.

2. CMS Collaboration. Multiplicity and rapidity dependence of strange hadron production in $\mathrm{pp}, \mathrm{pPb}$, and PbPb collisions at the LHC. Phys. Lett. B 2017, 768, 103-129, doi:10.1016/j.physletb.2017.01.075.

3. Gleisberg, T.; Hoeche, S.; Krauss, F.; Schönherr, M.; Schumann, S.; Siegert, F.; Winter, J. Event generation with SHERPA 1.1. JHEP 2009, 2009, 007.

4. Bellm, J.; Gieseke, S.; Grellscheid, D.; Plätzer, S.; Rauch, M.; Reuschle, C.; Richardson, P.; Schichtel, P.; Seymour, M.H.; Siódmok, A.; et al. Herwig 7.0/Herwig++ 3.0 release note. Eur. Phys. J. C 2016, 76, 196.

5. Sjöstrand, T.; Ask, S.; Christiansen, J.R.; Corke, R.; Desai, N.; Ilten, P.; Mrenna, S.; Prestel, S.; Rasmussen, C.O.; Skands, P.Z. An introduction to PYTHIA 8.2. Comput. Phys. Commun. 2015, 191, 159-177.

6. Pierog, T.; Karpenko, I.; Katzy, J.M.; Yatsenko, E.; Werner, K. EPOS LHC: Test of collective hadronization with data measured at the CERN Large Hadron Collider. Phys. Rev. C 2015, 92, 034906.

7. Lin, Z.-W.; Ko, C.M.; Li, B.-A.; Zhang, B.; Pal, S. Multiphase transport model for relativistic heavy ion collisions. Phys. Rev. C 2005, 72, 064901.

8. Wang, X.-N.; Gyulassy, M. HIJING: A Monte Carlo model for multiple jet production in pp, pA, and AA collisions. Phys. Rev. D 1991, 44, 3501.

9. Andersson, B.; Gustafson, G.; Nilsson-Almqvist, B. A model for low-pT hadronic reactions with generalizations to hadron-nucleus and nucleus-nucleus collisions. Nucl. Phys. B 1987, 281, 289-309.

10. $\mathrm{Pi}, \mathrm{H}$. An event generator for interactions between hadrons and nuclei-FRITIOF version 7.0. Comput. Phys. Commun. 1992, 71, 173-192.

11. Alvioli, M.; Strikman, M. Color fluctuation effects in proton-nucleus collisions. Phys. Lett. B 2013, 722, 347-354.

12. Bierlich, C.; Gustafson, G.; Lonnblad, L.; Shah, H. The Angantyr model for heavy-ion collisions in Pythia 8. JHEP 2018, 2018, 134.

13. Aad, G.; Abajyan T.; Abbott B.; Abdallah J.; Abdel Khalek S.; Abdinov O.; Aben R.; Abi B.; Abolins M.; AbouZeid O.S. et al. (ATLAS Collaboration). Measurement of the centrality dependence of the charged-particle pseudorapidity distribution in proton-lead collisions at $\sqrt{{ }_{S_{N N}}}=5.02 \mathrm{TeV}$ with the ATLAS detector. Eur. Phys. J. C 2016, 76, 199, arXiv:1508.00848.

14. Aamodt, K.; Abrahantes Quintana A.; Adamova D.; Adare A.M.; Aggarwal M.M.; Aglieri Rinella G.; Agocs A.G.; Aguilar Salazar S.; Ahammed Z.; Ahmad N. et al. (ALICE Collaboration). Centrality dependence of the charged-particle multiplicity density at mid-rapidity in $\mathrm{Pb}-\mathrm{Pb}$ collisions at $\sqrt{s_{N N}}=2.76 \mathrm{TeV}$ Phys. Rev. Lett. 2011, 106, 032301, arXiv:1012.1657. 
15. Acharya, S.; Acosta F.T.-.; Adamova D.; Adolfsson J.;Aggarwal M.M.; Aglieri Rinella G.; Agnello M.; Agrawal N.; Ahammed Z.; Ahn S.U. et al. (ALICE Collaboration). Centrality and pseudorapidity dependence of the charged-particle multiplicity density in Xe-Xe collisions at $\sqrt{s_{N N}}=5.44 \mathrm{TeV}$ arXiv:1805.04432.

(C) 2019 by the authors. Licensee MDPI, Basel, Switzerland. This article is an open access article distributed under the terms and conditions of the Creative Commons Attribution (CC BY) license (http:// creativecommons.org/licenses/by/4.0/). 